\title{
MicroRNA-27a promotes tumorigenesis via targeting AKT in triple negative breast cancer
}

\author{
JING WU, ZHIHUI SUN, HUIJIE SUN and YANHUA LI \\ Daytime Observation Ward, The First Hospital, Jilin University, Changchun, Jilin 130021, P.R. China
}

Received March 29, 2017; Accepted September 25, 2017

DOI: $10.3892 / \mathrm{mmr} .2017 .7886$

\begin{abstract}
Altered microRNA (miRNA/miR) expression regulates tumor development and progression in triple-negative breast cancer (TNBC). The present study examined the effect of miR-27a on proliferation, migration and invasion of TNBC cells in vitro and in vivo. An MTT assay was performed to examine the proliferation of MDA-MB-231 and MDA-MB-468 breast cancer cells with either overexpression of miR-27a or downregulation of miR-27a, in the presence or absence of radiation. The migratory and invasive abilities of MDA-MB-231 and MDA-MB-468 breast cancer cells were assessed by Transwell migration and Matrigel invasion assays. The protein expression levels were examined by western blotting. The caspase-Glo3/7 assay was performed to examine the effect of miR-27a on radiation-induced apoptosis in MDA-MB-231 and MDA-MB-468 breast cancer cells. A luciferase assay was performed to evaluate the effect of miR-27a on phosphatase and tensin homolog (PTEN) and B cell lymphoma ( $\mathrm{Bcl})-2$ associated $\mathrm{X}$, apoptosis regulator (BAX) expression. Immunodeficient nude mice were used to examine tumor growth following injection of MDA-MB-231 breast cancer cells. miR-27a promoted proliferation in vitro and in vivo, and enhanced migration and invasion in TNBC cells. miR-27a improved the survival of TNBC cells following irradiation. miR-27a inhibited radiation-induced apoptosis in TNBC cells by regulation of caspase 3/7 and Bcl-2 expression. Furthermore, the expression levels of PTEN and phosphorylated protein kinase B in MDA-MB-231 and MDA-MB-468 cells was altered following overexpression of miR-27a. The luciferase assay demonstrated that miR-27a regulated PTEN and BAX expression by binding to 3'-untranslated regions. Overall, miR-27a exhibits an essential role in tumor development and progression in TNBC and may be used as a potential biomarker to predict radiotherapy response and prognosis for the disease.
\end{abstract}

Correspondence to: Dr Yanhua Li, Daytime Observation Ward, The First Hospital, Jilin University, 71 Xinmin Avenue, Changchun, Jilin 130021, P.R. China

E-mail: yanhua.li2@aol.com

Key words: miR-27a, triple negative breast cancer, apoptosis, AKT, PTEN

\section{Introduction}

Breast cancer remained the leading cause of cancer death in women although mortality is falling due to earlier diagnosis and improved surgical techniques as well as better chemotherapy and radiotherapy (1-3). It is estimated that about 252,710 new cases of invasive breast cancer will be diagnosed in women, and about 40,610 women will die from breast cancer in 2017 in the United States (4).

About 15-20\% of breast cancers are found to be triple-negative for estrogen receptors, progesterone receptors, and HER2, and defined as triple-negative breast cancer (TNBC) (5). TNBC has a poor prognosis and only responds to chemotherapy and radiotherapy but not hormonal therapy $(6,7)$. Therefore, discovery of new molecular targets to treat patients with TNBC has been pressing and of significant interest $(8,9)$.

MicroRNAs (miRNAs/miRs) are small non-coding RNAs that play important roles in regulation of gene expression post-transcriptionally (10). Growing evidence has shown that some miRNAs are upregulated in cancer and behave as oncogenic characteristics, while some miRNAs are downregulated in cancer and act as tumor-suppressive miRNAs. Therefore, miRNAs play critical roles in each stages of tumorigenesis of many human cancers such as lung (11), endometrial (12), and colon cancer (13). miRNAs are promising potential targets for cancer treatment. Previous studies have shown that miRNAs are associated with different biological activities in breast cancer. Huang et al showed that miR-21 improved breast cancer cell invasion and regulated epithelial-to-mesenchymal transition (EMT) (14). Overexpression of miR-205 decreases cell proliferation and increases apoptosis via regulation of HMGB3 in breast cancer. miR-205 regulates HMGB3 and its ectopic expression significantly inhibits cell proliferation and promotes apoptosis in breast cancer (15). Recently, Liu et al reported that aberrant expression of miR-374b-5p, miR-218-5p, miR-126-3p, miR-27b-3p predicted a good prognosis in TNBC (16).

In this study, we examined the function of miR-27a in cell proliferation, invasion and migration using TNBC cell lines.

\section{Materials and methods}

Cell lines. MDA-MB-231 and MDA-MB-468, human TNBC cell lines were purchased from American Type Culture Collection (ATCC, Manassas, VA, USA). The tumor cells 
were grown in Dubelcco's modified Egale's medium (DMEM) (Invitrogen, Carlsbad, CA, USA) supplemented with $100 \mu \mathrm{g} / \mathrm{ml}$ streptomycin, $100 \mathrm{U} / \mathrm{ml}$ penicillin and $10 \%$ fetal bovine serum (Invitrogen), and at $37^{\circ} \mathrm{C}$ in a humidified incubator with $5 \% \mathrm{CO}_{2}$. When all cells reach to $70-80 \%$ confluence, they were used in our experiments.

Cell transfection and miRNA quantification. To transfer miR-27a mimics or anti-miR-27a inhibitor (Invitrogen) into breast cancer cells, Lipofectamine 2000 (Invitrogen) was used. A random sequence miRNA mimic molecule was used as a negative control (mirVana ${ }^{\mathrm{TM}}$ miRNA mimic; Ambion, Austin, TX, USA). Then, miR-27a expression level after transfection was examined. The total RNA was extracted from the transfected breast cancer cells, TaqMan miRNA reverse transcription kit (Thermo Fisher Scientific, Inc., Waltham, MA, USA) was used to synthesize cDNA according the manufacturer's instructions. The GAPDH, one of housekeeping genes, was used as the endogenous reference gene.

Cell proliferation assay. To analyze the effect of miR-27a on breast cancer cell proliferation, WST-1 assay (Roche Diagnostics, Indianapolis, IN, USA) was performed. Briefly, the transfected breast cancer cells were placed into 96-well plates at the density of $2 \times 10^{4}$ cells/well and cultured overnight. The cells were continued to culture at $37^{\circ} \mathrm{C}$ in a humidified incubator with $5 \% \mathrm{CO}_{2} .20 \mu \mathrm{l}$ of WST-1 reagent was added to each well and incubated for at least $1 \mathrm{~h}$ at $37^{\circ} \mathrm{C}$ every $24 \mathrm{~h}$. Then the absorbance was measured at $490 \mathrm{~nm}$. All experiments were performed in triplicates.

Migration and invasion assays. To evaluate breast cancer cell migration and invasion, the Promega migration and invasion assays were performed according the manufacturer's instructions. Briefly, transfected breast cancer cells were seeded on the upper Transwell chamber either with or without Matrigel in DMEM without FBS. DMEM containing 5\% FBS was added to the lower chamber. The cells were cultured for $18 \mathrm{~h}$ at $37^{\circ} \mathrm{C}$. Then the non-invaded cells were removed by cotton swabs, and the invaded cells were stained by Diff-Quik stain. The percentage of migration and invasion was calculated and showed as a ratio of invaded cells over cells normalized on second day of growth curve.

Western blot analysis. The breast cancer cells were gently washed with cold phosphate-buffered saline, and then lysed in ice-cold lysis buffer (50 mM Tris- $\mathrm{HCl}, \mathrm{pH} 7.5,0.1 \%$ SDS, $150 \mathrm{mM} \mathrm{NaCl}, 0.5 \%$ deoxycholate, $1 \% \mathrm{NP}-40$, and $1 \mathrm{X}$ protease inhibitors), then heated at $100^{\circ} \mathrm{C}$ for $5 \mathrm{~min}$. Protein lysates $(20 \mu \mathrm{g})$ were loaded to the SDS-PAGE gel and transferred to PVDF membranes (Sigma-Aldrich, St. Louis, MO, USA). The PVDF membranes were incubated in blocking buffer for $1 \mathrm{~h}$ at room temperature. Different primary antibodies [B cell lymphoma (Bcl)-2, Bcl-2 associated X (BAX), AKT and p-AKT; Cell Signaling Technology, Inc., Danvers, MA, USA] were added in 5\% non-fat dry milk in TBS-T buffer at $4^{\circ} \mathrm{C}$ overnight, followed by secondary antibody incubation $1 \mathrm{~h}$ at room temperature. The immune signals were detected with the EasySee Weatern Blot kit (TransGen Biotech Co., Ltd., Shanghai, China).
Apoptosis activity after ionizing radiation treatment. To examine the apoptosis activity of breast cancer cells with altered miR-27a expression, the breast cancer cells were grown in 24-well plates at density of $2 \times 10^{5} /$ well and cultured overnight. Then the breast cancer cells were exposed to different doses of ionizing radiation. After $24 \mathrm{~h}$ of culture, the apoptosis activity was examined by measure the caspase $3 / 7$ activity using the Caspase-Glo3/7 assay kit (Promega Corp., Madison, WI, USA) following the manufacturer's protocol. Briefly, Caspase-Glo reagent was added to each well, and then the cells were incubated with the Caspase-Glo reagent in a dark place for $8 \mathrm{~h}$ with gentle shaking at room temperature. The luminescence value was measured using 1-min lag time and $0.5 \mathrm{sec} /$ well read time. All experiments were carried out in triplicates.

Luciferase reporter assay. The pEZX-MT05 reporter vector carrying miRNA full length binding sequence of 3'-UTR of phosphatase and tensin homolog (PTEN) gene, BAX or control sequence (GeneCopoeia, Rockville, MD, USA) was co-transfected to HEK-293T cells with has-miR-27a using Lipofectamine 2000 (Invitrogen). Two days after the transfections, the culture medium was collected and Gaussian luciferase and alkaline phosphatase activities were measured using the secreted pair dual luminescence kit according to the manufacturer's instructions (GeneCopoeia). Gaussian luciferase activity was normalized to alkaline phosphatase activity.

Xenograft assays in nude mice. Female BALB/c athymic nude mice (5-week-old) purchased from Charles River Laboratories (Wilmington, MA, USA) were used in xenograft assay. The mice were maintained in accordance with the Guide for Institutional Animal Care and guidelines for animal experiment. MDA-MB-231 breast cancer cells $\left(1 \times 10^{6}\right)$ with or without miR-27a mimics were mixed with Matrigel ECM (reconstituted basement membrane) (17) and were injected subcutaneously into the mammary fat pads. Ten mice were included in each experimental group. Tumor size was measured every 3 days by measuring tumor length (L) and width (W) with calipers, and tumor volume was calculated as: Tumor volume $=\pi \mathrm{LW}^{2} / 6(18)$.

Statistical analysis. All of results in our experiments were shown as Mean \pm standard deviation. SPSS program (version 11.0; SPSS, Inc., Chicago, IL, USA) was chose for statistical analyses using Student's t-test. Differences are considered statistically significant if $\mathrm{P}<0.05$.

\section{Results}

miR-27a improved the proliferation of TNBC cells both in vitro and in vivo. To determine the effect of miR-27a on proliferation of TNBC cells, miR-27a was transfected to MDA-MB-231 and MDA-MB-468 TNBC cells with Lipofectamine 2000. MTT assay was performed to examine the growth curve (Fig. 1). As shown in Fig. 1A, the miR-27a expression level was significantly increased in both MDA-MB-231 and MDA-MB-468 cells after transfection of miR-27a mimic, and the miR-27a expression level was decreased after transfection of anti- miR-27a inhibitor. miR-27a promoted the proliferation of MDA-MB-231 
A
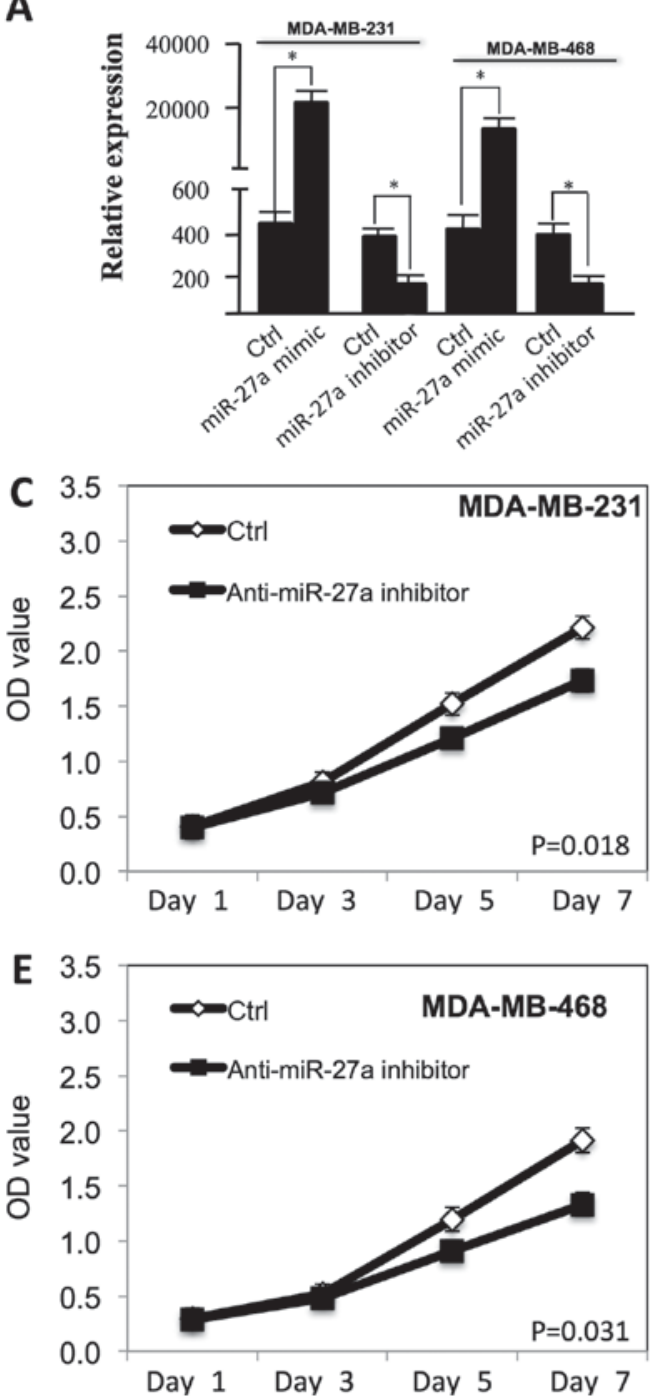

G

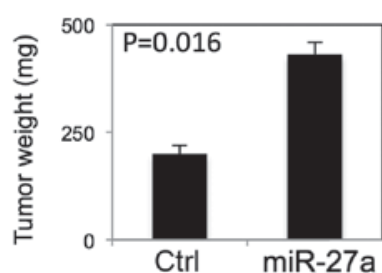

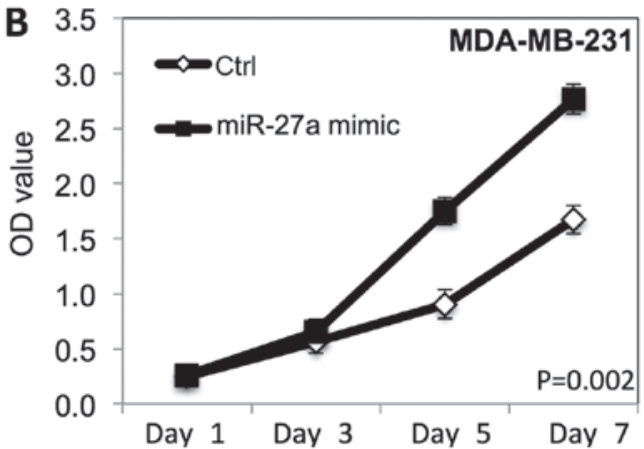
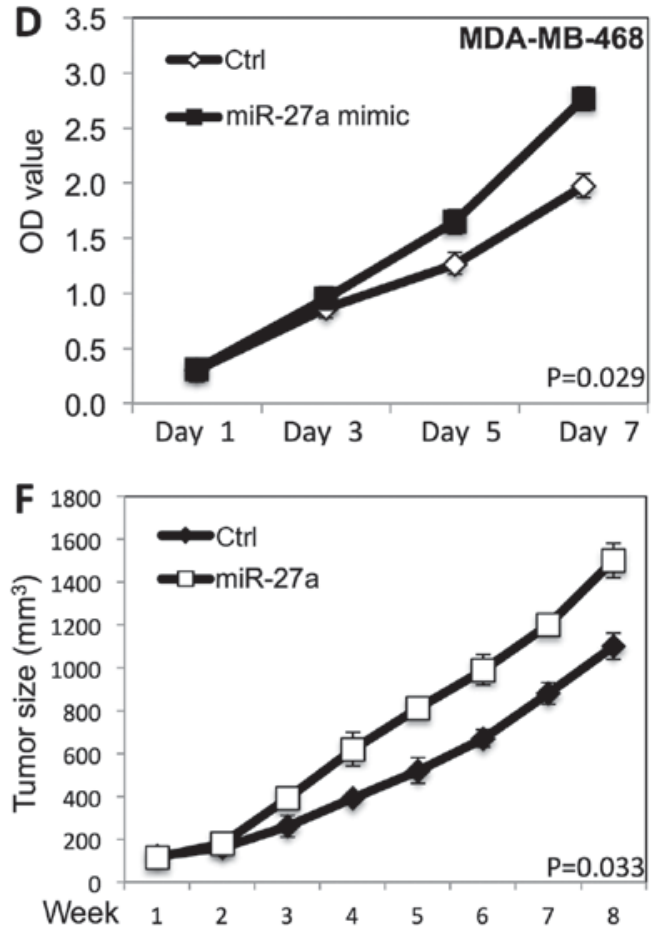

H

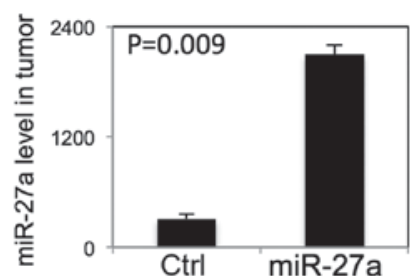

Figure 1. miR-27a promoted the proliferation of TNBC cells. (A) The miR-27a expression level was determined by qRT-PCR in TNBC cells transfected with either miR-27a mimic or miR-27a inhibitor. $\mathrm{P}=0.002, \mathrm{P}=0.008, \mathrm{P}=0.006, \mathrm{P}=0.004$. (B) The proliferation of MDA-MB-231 cells after miR-27a overexpression. (C) The proliferation of MDA-MB-231 cells after transfection with anti-miR-27a inhibitor. (D) The proliferation of MDA-MB-468 cells after miR-27a overexpression. (E) The proliferation of MDA-MB-468 cells after transfection with anti-miR-27a inhibitor. (F) The in vivo growth of MDA-MB-231 cells transfected with miR-27a mimic in nude mice exnografts. (G) The average tumor weight in nude mice exnografts. (H) The miR-27a expression level in nude mice exongrafts. ${ }^{*} \mathrm{P}<0.05$. miR, microRNA; TNBC, triple-negative breast cancer.

(Fig. 1B) and MDA-MB-468 cells (Fig. 1D) $(\mathrm{P}<0.05)$. When the expression level of miR-27a was decreased by the anti-miR-27a inhibitor, the proliferation of MDA-MB-231 (Fig. 1C) and MDA-MB-468 cells (Fig. 1E) was inhibited $(\mathrm{P}<0.05)$.

To examine whether miR-27a regulates tumor growth in vivo, we performed subcutaneous tumor xenograft experiments by injection transfected MDA-MB-231 breast cancer cells into immunodeficient nude mice. Compared to vector control group, the tumor growth rate and tumor weight in transfected MDA-MB-231 xenografts was significantly increased $(\mathrm{P}<0.01)$ (Fig. $1 \mathrm{~F}$ and $\mathrm{G})$. These in vivo results are consistent with the in vitro experiment results, and show miR-27a improved the tumor growth of breast cancer cells in vivo. The miR-27a expression level is high in tumor with transfected MDA-MB-231 cells (Fig. 1H).

miR-27a enhanced migration and invasion of TNBC cells. To examine the effect of miR-27a on migration and invasion of TNBC cells, we performed the migration and Matrigel invasion assays using BD Transwell. As shown in Fig. 2, the migration and invasion in MDA-MB-231 (Fig. 2A-D) and MDA-MB-468 (Fig. 2I-L) were significantly increased after 

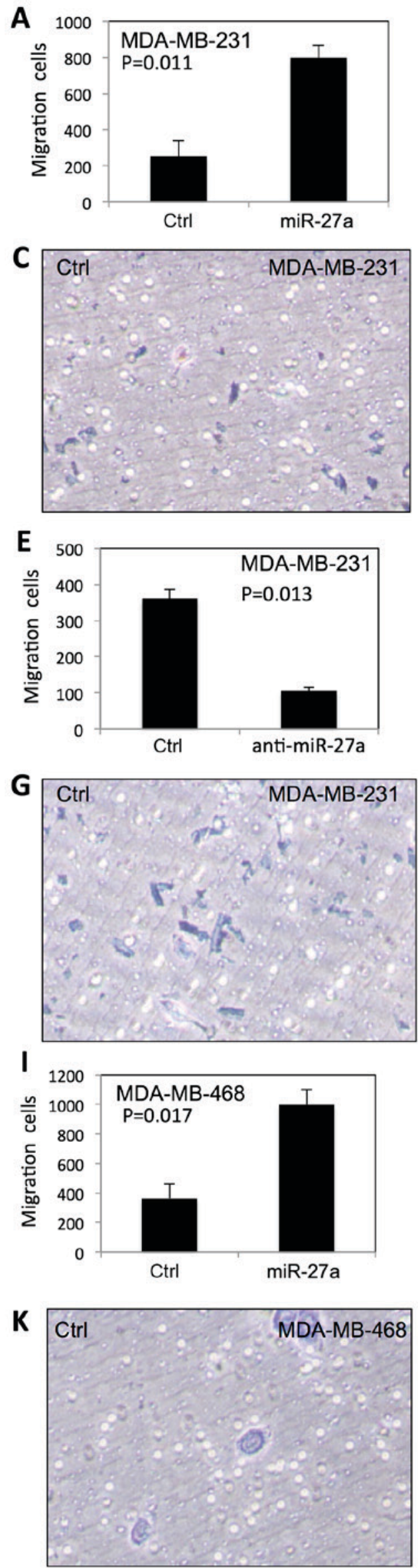

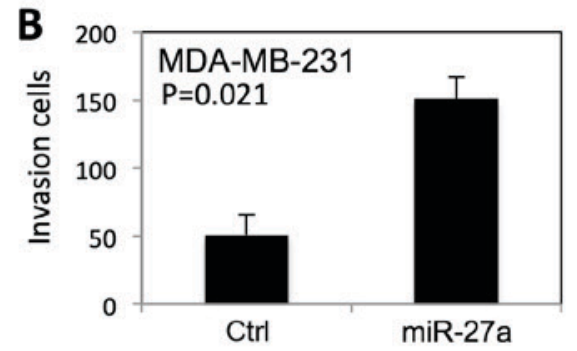

D
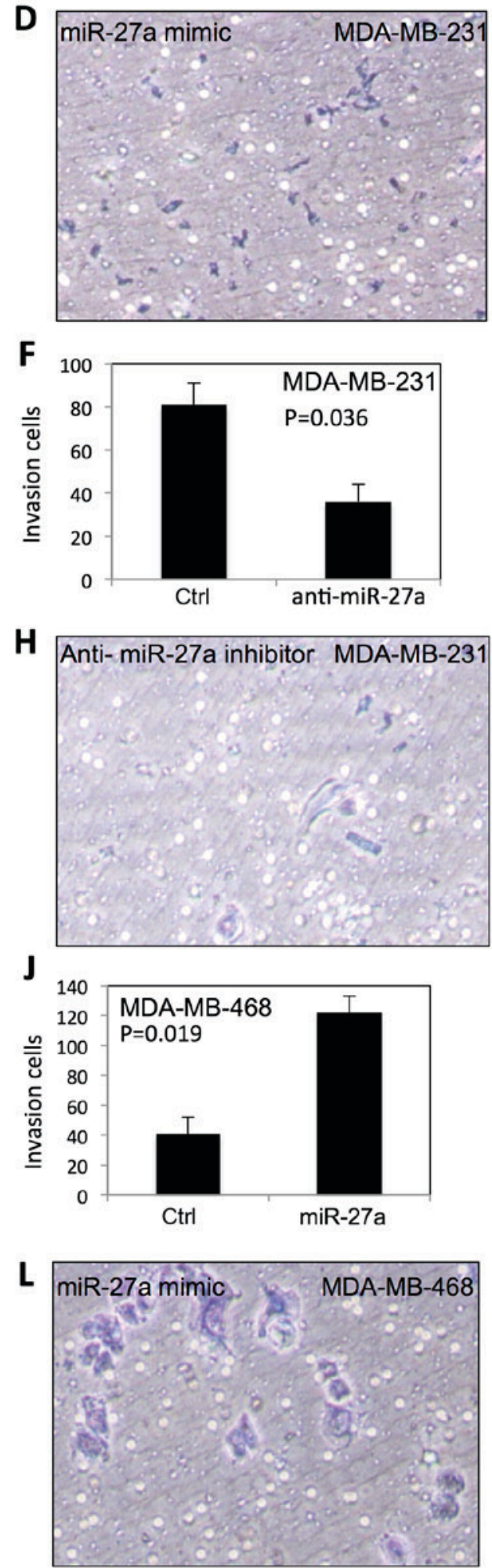

Figure 2. miR-27a increases migration and invasion of TNBC cells. (A-D) miR-27a increases migration and invasion of MDA-MB-231 cells. (E-H) The migration and invasion of MDA-MB-231 cells were decreased after treatment with anti-miR-27a inhibitor. (I-L) miR-27a increases migration and invasion in MDA-MB-468 cells 

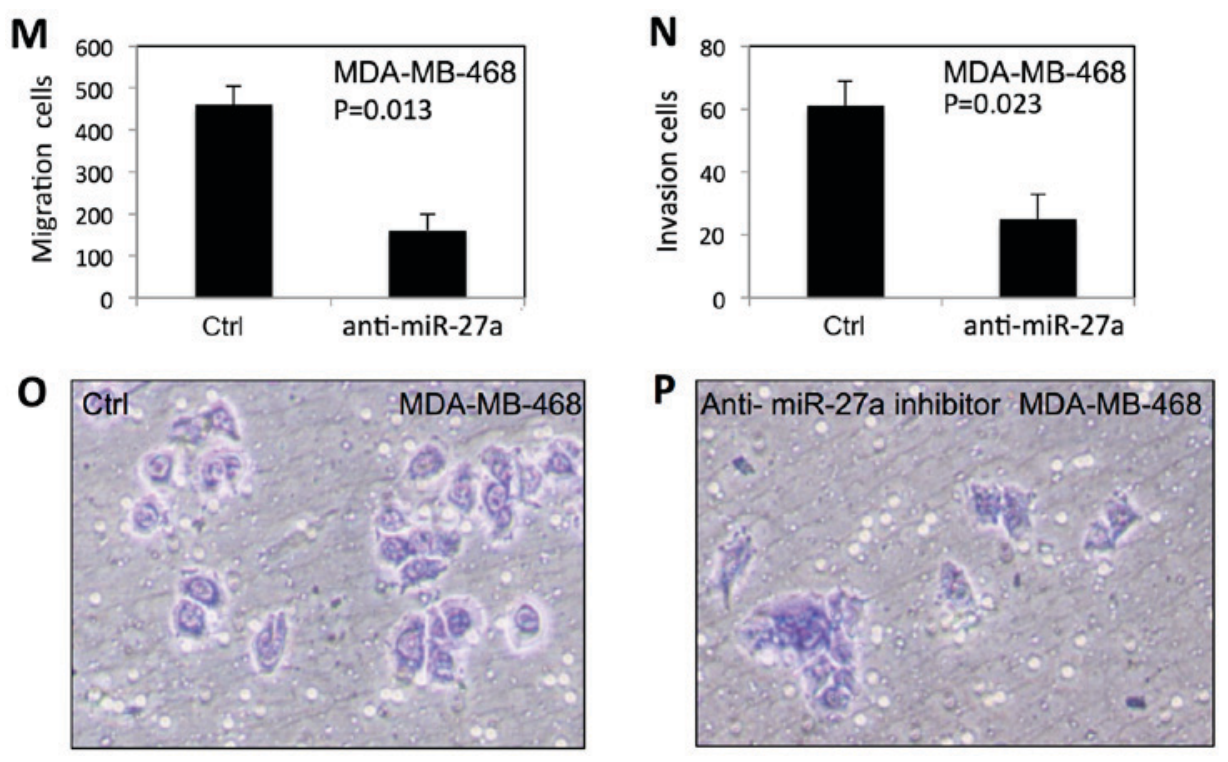

Figure 2. Continued. (M-P) The migration and invasion of MDA-MB-468 cells were decreased after treatment with anti-miR-27a inhibitor. All experiments were performed three times in triplicate. miR, microRNA.
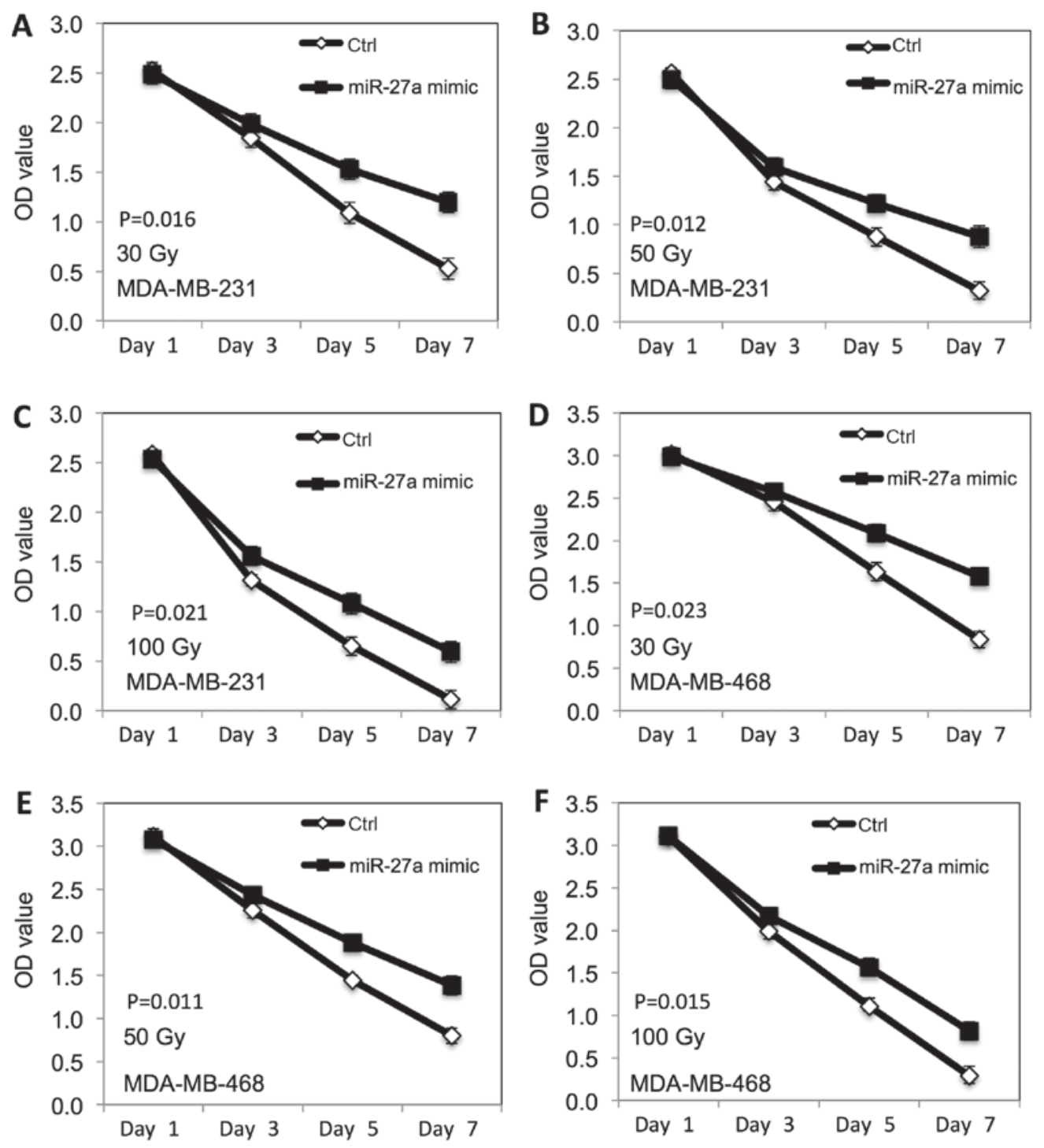

Figure 3. miR-27a improved the survival of TNBC cells after irradiation. (A-C) The effect of miR-27a on proliferation of MDA-MB-231 cells after different doses of irradiation treatment. (D-F) The effect of miR-27a on proliferation of MDA-MB-468 cells after different doses of irradiation treatment. miR, microRNA; TNBC, triple-negative breast cancer. 
A

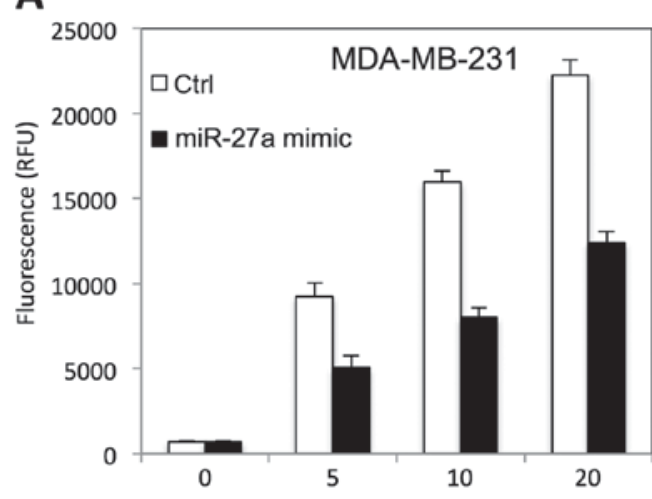

C

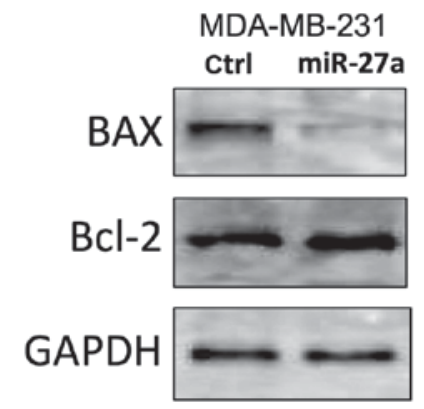

E

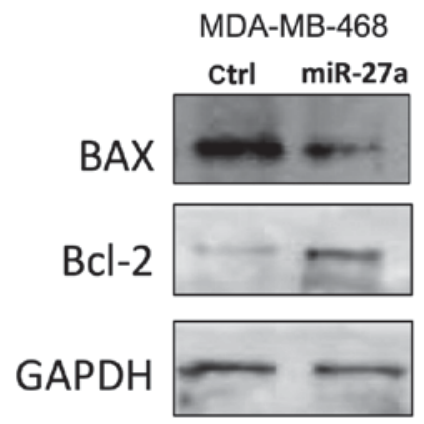

B

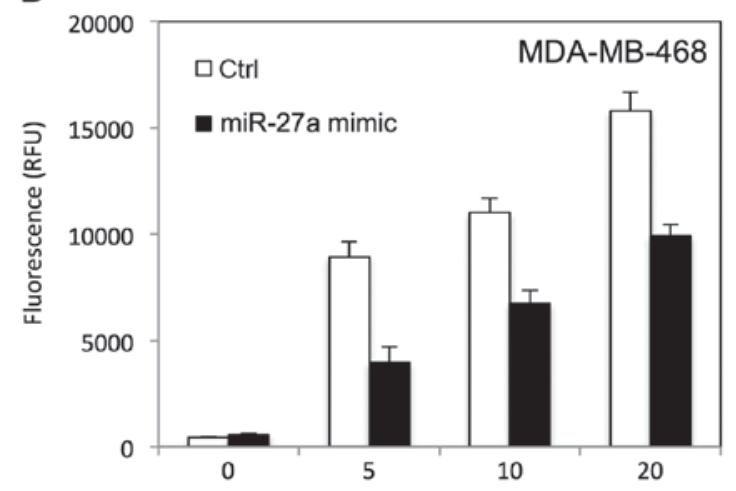

D

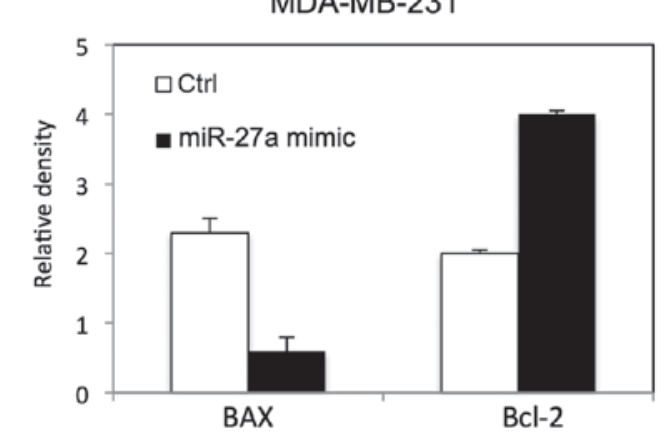

F

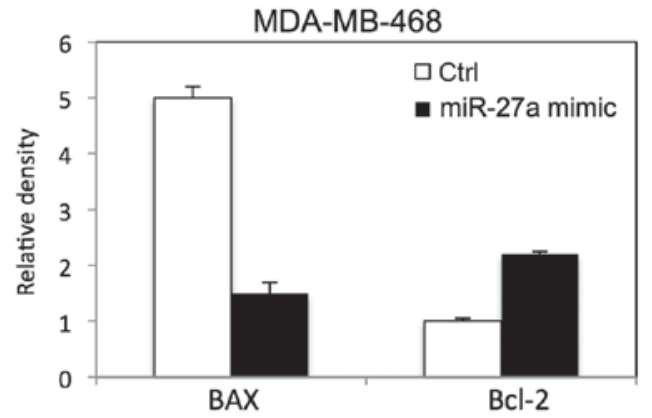

Figure 4. miR-27a inhibits radiation-induced apoptosis in TNBC cells. (A) The caspase 3/7 activity in MDA-MB-231 cells transfected with miR-27a after different doses of irradiation treatment. (B) The caspase 3/7 activity in MDA-MB-468 cells transfected with miR-27a after different doses of irradiation treatment. (C and D) The apoptotic proteins levels in MDA-MB-231 cells after radiation. (E and F) The apoptotic proteins levels in MDA-MB-468 cells after radiation. miR, microRNA; TNBC, triple-negative breast cancer; Bcl, B cell lymphoma; BAX, B cell lymphoma-2 associated X.

transfection of miR-27a. In contrast, the migration and invasion of MDA-MB-231 (Fig. 2E-H) and MDA-MB-468 cells (Fig. 2M-P) were significantly decreased by anti-miR-27a inhibitor.

miR-27a altered the radiation-induced inhibitory effect on proliferation of TNBC cells. We further examined the radiation-induced inhibitory effect of miR-27a on the proliferation of TNBC cells. The MDA-MB-231 and MDA-MB-468 breast cancer cells transfected with miR-27a were exposed to different doses of ionizing radiation (30,50 and $100 \mathrm{~Gy})$. MTT assay was performed to evaluate the proliferation. As shown in Fig. 3, the radiation-induced inhibitory effect on proliferation of both MDA-MB-231 (Fig. 3A-C) and MDA-MB-468 (Fig. 3D-F) cells was decreased at dose dependent manner after miR-27a overexpression, compared to the control cells.

miR-27a decreased radiation-induced apoptosis in TNBC cells. To determine the role of miR-27a on radiation-induced apoptosis in TNBC cells by measuring caspase 3/7 activity, and apoptotic related protein. As shown in Fig. 4, miR-27a inhibited the caspase 3/7 activity in both MDA-MB-231 (Fig. 4A) and MDA-MB-468 (Fig. 4B) cells at dose dependent manner. The expression of BAX and Bcl-2 was altered in MDA-MB-231 (Fig. 4C and D) and MDA-MB-468 (Fig. 4E and F) after miR-27a overexpression, compared to the control cells.

miR-27a activated AKT in TNBC cells. We further examined the expression of $\mathrm{p}-\mathrm{AKT}$, total AKT, and PTEN in both MDA-MB-231 and MDA-MB-468 cells after miR-27a overexpression. We found that miR-27a increased the expression of p-AKT, and decreased the PTEN expression level in both MDA-MB-231 (Fig. 5A and B) and MDA-MB-468 (Fig. 5C and D) cells.

To investigate whether miR-27a is able to regulate PTEN and BAX expression directly, we searched the miR-27a potential binding sites within the PTEN 3'-UTR and BAX 3'-UTR by 
A

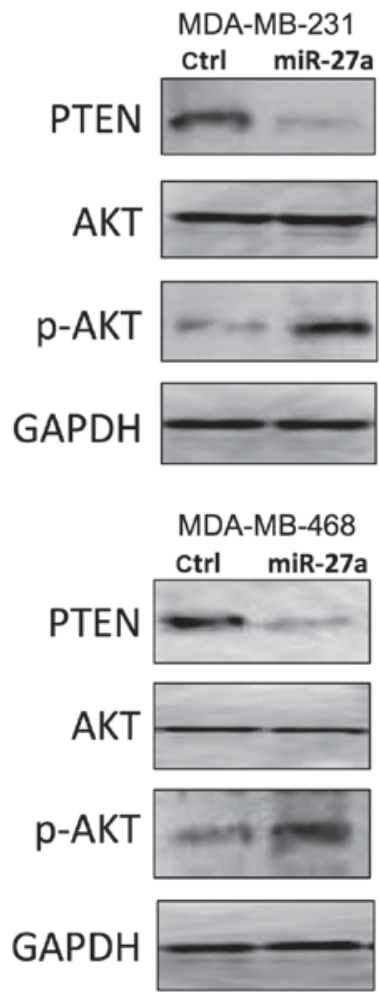

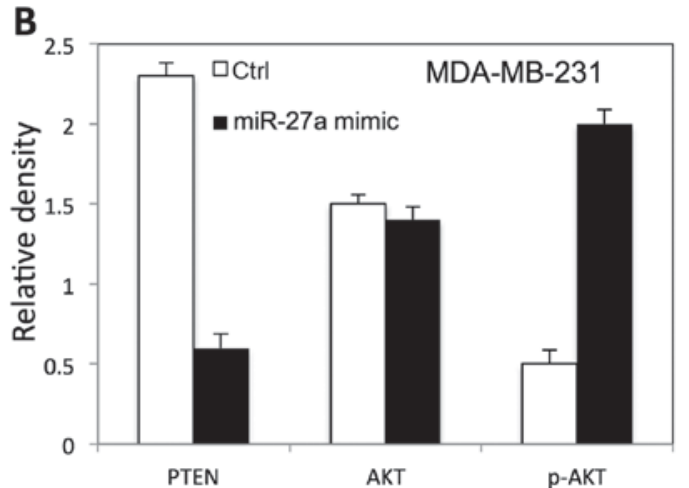

D

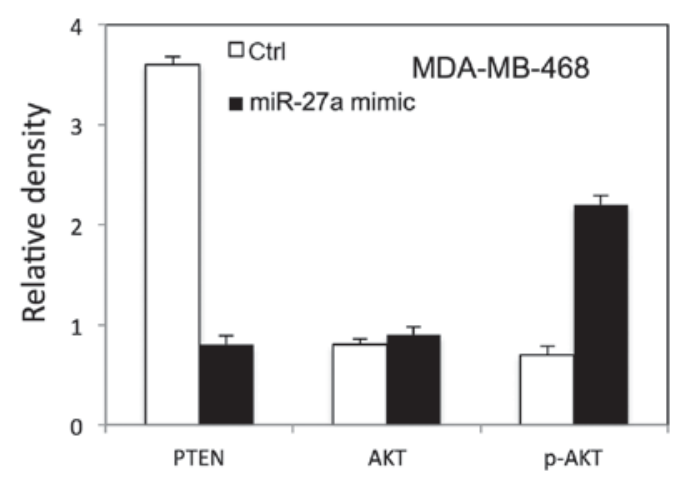

$\mathbf{F}$
E

3' cgccuUgaAUC-GGUGAC-ACUu 5' hsa-miR-27a

5' cgauaAuguagCCCACUgUUGac 3' PTEN

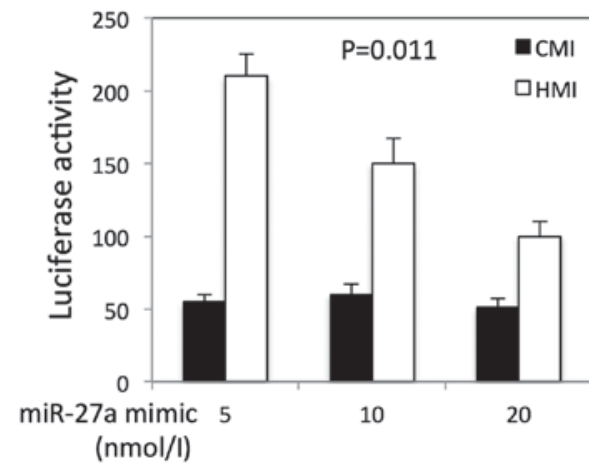

$3^{\prime}$ cgccuugaAuCG-gugACACUu 5' hsa-miR-27a

5' ugaccCCUGACCUCACUGUGAC 3' BAX

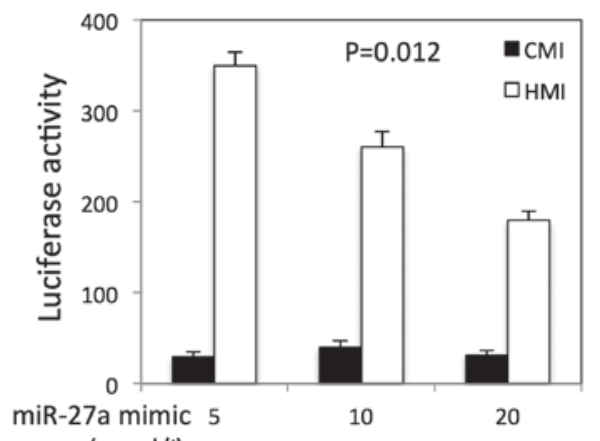

(nmol/l)

Figure 5. miR-27a activated AKT in TNBC cells. (A) The expression of, p-AKT, AKT and PTEN in MDA-MB-231 cells after transfection with miR-27a mimic. (B) The relative expression level of proteins on MDA-MB-231 cells after transfection with miR-27a mimic by densitometric analysis. (C) The expression of p-AKT, AKT and PTEN in MDA-MB-468 cells after transfection with miR-27a mimic. (D) The relative expression level of proteins on MDA-MB-468 cells after transfection with miR-27a mimic by densitometric analysis. (E and F) The potential binding sequence between miR-27a and target genes (PTEN and BAX) and luciferase activity after transfection of miR-27a in dose-dependent manner with luciferase reporter containing PTEN and BAX 3'-UTR. miR, microRNA; TNBC, triple-negative breast cancer; PTEN, phosphatase and tensin homolog; Bax, B cell lymphoma-2 associated X.

miRNA target gene prediction software (TargetScanHuman). The search showed that miR-27a has a potential binding site within the PTEN 3'-UTR and BAX 3'-UTR (Fig. 5E and F).

To evaluate the effect of miR-27a on PTEN and BAX expression, we performed luciferase assay with a reporter (HMI) containing 3'-UTR of PTEN and BAX gene. The luciferase activity of the construct (HMI) significantly increased in a dosage-dependent manner by co-transfection of miR-27a mimic in a dosage-dependent manner (Fig. 5E and F). However, miR-27a transfection had no effect on luciferase activity in control plasmid (CMI) lacking the PTEN 3'-UTR fusion (Fig. 5E and F).

\section{Discussion}

Recent studies have shown that miR-27a plays important roles in tumorgenesis in many organs, including kidney (19), breast (20), stomach (21) and cervix (22). For instance, Pan et al found that miR-27a improved proliferation, migration and invasion of human osteosarcoma cells by regulating MAP2K4 expression (23). The overexpression of miR-27a is associated with metastasis in gastric cancer cells by regulating EMT (24). It has been demonstrated that Wnt/ $\beta$-catenin signaling pathway is associated with the proliferation and migration of breast cancer. Kong et al found that miR-27a 
promoted proliferation of breast cancer cells by targeting SFRP1 via Wnt/ $\beta$-catenin signaling pathway both in vitro and in vivo (25). In addition, Drayton et al reported that miR-27a contributed to cisplatin resistance by targeting cysteine/glutamate exchanger SLC7A11 (26). Recently study has shown that miR-27a might act as a prognostic marker for breast cancer (27). Furthermore, miR-27a may coordinate with other miRNAs in breast cancer cells (28). In our study, we found that miR-27a improved the proliferation of TNBC cells. Moreover, we showed that overexpression of miR-27a enhanced migration and invasion in both MDA-MB-231 and MDA-MB-468 cells. However, downregulation of miR-27a decreased the migration and invasion in both MDA-MB-231 and MDA-MB-468 cells. It has been known that TNBC is more likely to recur and has poor prognosis (29). Metastasis is one of important cause for the recurrence of TNBC. Therefore, our study indicated that miR-27a play important roles in TNBC progression.

Radiotherapy is one of highly effective adjuvant treatments in patients with breast cancer after surgery (30). TNBC is characterized as rapid growth and local recurrence. It has been found that radiotherapy can decrease the locoregional recurrence in patients with T1-2N0 disease after modified radical mastectomy (31). In the present study, we found that miR-27a enhanced the survival of TNBC cells after irradiation. Meanwhile, miR-27a inhibited the radiation-induced apoptosis of TNBC cells.

PTEN, the second most frequently mutated tumor suppressor gene in human cancer, plays critical roles in proliferation, apoptosis and cell cycle in tumor cells. PTEN involves tumor development by targeting several signaling pathways, such as MAPK pathway, FAK pathway and PI3K/AKT pathway. Recent studies have demonstrated that PI3K/AKT pathway is the key pathway by which PTEN displays antioncogenic effects (32). The PI3K/AKT pathway regulates multiple biological processes and mediates the downstream responses including cell proliferation, apoptosis and metabolism. It has been found that PI3K/AKT is activated in TNBC due to PTEN loss (33). Our results showed that PTEN expression level was decreased, and p-AKT expression level was increased in TNBC cells after overexpression miR-27a. In addition, luciferase assay showed that PTEN and BAX are downregulated by miR-27a by binding to 3 '-UTR. These results indicated miR-27a regulates proliferation of TNBC cells by targeting PI3K/AKT signaling pathway. Further studies are needed to demonstrate the molecular mechanism.

miR-27a regulated tumorigenesis and malignant progression of TNBC. The present study indicates miR-27a might be used as a potential biomarker to predict the radiotherapy response and prognosis in TNBC.

\section{References}

1. Siegel RL, Miller KD and Jemal A: Cancer statistics, 2016. CA Cancer J Clin 66: 7-30, 2016.

2. Webb PM, Cummings MC, Bain CJ and Furnival CM: Changes in survival after breast cancer: Improvements in diagnosis or treatment? Breast 13: 7-14, 2004.

3. Di Leo A, Curigliano G, Diéras V, Malorni L, Sotiriou C, Swanton C, Thompson A, Tutt A and Piccart M: New approaches for improving outcomes in breast cancer in Europe. Breast 24: 321-330, 2015
4. American Cancer Society: Breast Cancer. http://www.cancer. org/cancer/breastcancer/detailedguide/breast-cancer-key-statistics.

5. Boyle P: Triple-negative breast cancer: Epidemiological considerations and recommendations. Ann Oncol 23 (Suppl 6): vi7-vi12, 2012.

6. Negi P, Kingsley PA, Jain K, Sachdeva J, Srivastava H, Marcus S and Pannu A: Survival of triple negative versus triple positive breast cancers: Comparison and contrast. Asian Pac J Cancer Prev 17: 3911-3916, 2016.

7. Braicu C, Chiorean R, Irimie A, Chira S, Tomuleasa C, Neagoe E, Paradiso A, Achimas-Cadariu P, Lazar V and Berindan-Neagoe I: Novel insight into triple-negative breast cancers, the emerging role of angiogenesis, and antiangiogenic therapy. Expert Rev Mol Med 18: e18, 2016

8. Linklater ES, Tovar EA, Essenburg CJ, Turner L, Madaj Z, Winn ME, Melnik MK, Korkaya H, Maroun CR, Christensen JG, et al: Targeting MET and EGFR crosstalk signaling in triple-negative breast cancers. Oncotarget 7: 69903-69915, 2016.

9. Gray MJ, Gong J, Hatch MM, Nguyen V, Hughes CC, Hutchins JT and Freimark BD: Phosphatidylserine-targeting antibodies augment the anti-tumorigenic activity of anti-PD-1 therapy by enhancing immune activation and downregulating pro-oncogenic factors induced by T-cell checkpoint inhibition in murine triple-negative breast cancers. Breast Cancer Res 18: 50, 2016.

10. Krol J, Loedige I and Filipowicz W: The widespread regulation of microRNA biogenesis, function and decay. Nat Rev Genet 11: 597-610, 2010.

11. Xue J, Yang J, Luo M, Cho WC and Liu X: MicroRNA-targeted therapeutics for lung cancer treatment. Expert Opin Drug Discov 12: 141-157, 2017.

12. Canlorbe G, Wang Z, Laas E, Bendifallah S, Castela M, Lefevre M, Chabbert-Buffet N, Daraï E, Aractingi S, Méhats C and Ballester M: Identification of microRNA expression profile related to lymph node status in women with early-stage grade 1-2 endometrial cancer. Mod Pathol 29: 391-401, 2016.

13. Mullany LE, Herrick JS, Wolff RK, Buas MF and Slattery ML: Impact of polymorphisms in microRNA biogenesis genes on colon cancer risk and microRNA expression levels: A population-based, case-control study. BMC Med Genomics 9: 21, 2016.

14. Huang TH, Wu F, Loeb GB, Hsu R, Heidersbach A, Brincat A, Horiuchi D, Lebbink RJ, Mo YY, Goga A and McManus MT: Up-regulation of miR-21 by HER $2 /$ neu signaling promotes cell invasion. J Biol Chem 284: 18515-18524, 2009.

15. Liu J, Mao Q, Liu Y, Hao X, Zhang S and Zhang J: Analysis of miR-205 and miR-155 expression in the blood of breast cancer patients. Chin J Cancer Res 25: 46-54, 2013.

16. Liu Y, Cai Q, Bao PP, Su Y, Cai H, Wu J, Ye F, Guo X, Zheng W, Zheng Y and Shu XO: Tumor tissue microRNA expression in association with triple-negative breast cancer outcomes. Breast Cancer Res Treat 152: 183-191, 2015.

17. Peng Y, Chen F, Melamed J, Chiriboga L, Wei J, Kong X, McLeod M, Li Y, Li CX, Feng A, et al: Distinct nuclear and cytoplasmic functions of androgen receptor cofactor p44 and association with androgen-independent prostate cancer. Proc Natl Acad Sci USA 105: 5236-5241, 2008.

18. Minn AJ, Gupta GP, Siegel PM, Bos PD, Shu W, Giri DD, Viale A, Olshen AB, Gerald WL and Massagué J: Genes that mediate breast cancer metastasis to lung. Nature 436: 518-524, 2005.

19. Gottardo F, Liu CG, Ferracin M, Calin GA, Fassan M, Bassi P, Sevignani C, Byrne D, Negrini M, Pagano F, et al: Micro-RNA profiling in kidney and bladder cancers. Urol Oncol 25: 387-392, 2007.

20. Mertens-Talcott SU, Chintharlapalli S, Li X and Safe S: The oncogenic microRNA-27a targets genes that regulate specificity protein transcription factors and the G2-M checkpoint in MDA-MB-231 breast cancer cells. Cancer Res 67: 11001-11011, 2007.

21. Liu T, Tang H, Lang Y, Liu M and Li X: MicroRNA-27a functions as an oncogene in gastric adenocarcinoma by targeting prohibitin. Cancer Lett 273: 233-242, 2009.

22. Wang X, Tang S, Le SY, Lu R, Rader JS, Meyers C and Zheng ZM: Aberrant expression of oncogenic and tumor-suppressive microRNAs in cervical cancer is required for cancer cell growth. PLoS One 3: e2557, 2008.

23. Pan W, Wang H, Jianwei R and Ye Z: MicroRNA-27a promotes proliferation, migration and invasion by targeting MAP2K4 in human osteosarcoma cells. Cell Physiol Biochem 33: 402-412, 2014.

24. Zhang Z,Liu S, Shi R and Zhao G: miR-27 promotes human gastric cancer cell metastasis by inducing epithelial-to-mesenchymal transition. Cancer Genet 204: 486-491, 2011. 
25. Kong LY, Xue M,Zhang QC and Su CF: In vivo and in vitro effects of microRNA-27a on proliferation, migration and invasion of breast cancer cells through targeting of SFRP1 gene via Wnt/ $\beta$-catenin signaling pathway. Oncotarget 8: 15507-15519, 2017.

26. Drayton RM, Dudziec E, Peter S, Bertz S, Hartmann A, Bryant HE and Catto JW: Reduced expression of miRNA-27a modulates cisplatin resistance in bladder cancer by targeting the cystine/glutamate exchanger SLC7A11. Clin Cancer Res 20: 1990-2000, 2014.

27. Tang W, Zhu J, Su S, Wu W, Liu Q, Su F and Yu F: MiR-27 as a prognostic marker for breast cancer progression and patient survival. PLoS One 7: e51702, 2012.

28. Guttilla IK and White BA: Coordinate regulation of FOXO1 by miR-27a, miR-96, and miR-182 in breast cancer cells. J Biol Chem 284: 23204-23216, 2009.

29. Dent R, Hanna WM, Trudeau M, Rawlinson E, Sun P and Narod SA: Pattern of metastatic spread in triple-negative breast cancer. Breast Cancer Res Treat 115: 423-428, 2009.

30. PDQ Pediatric Treatment Editorial Board: Childhood craniopharyngioma treatment $\left(\mathrm{PDQ}^{\circledR}\right)$ : Health professional version. In: PDQ Cancer Information Summaries. National Cancer Institute (US), Bethesda, MD, 2002.
31. Abdulkarim BS, Cuartero J, Hanson J, Deschênes J, Lesniak D and Sabri S: Increased risk of locoregional recurrence for women with T1-2N0 triple-negative breast cancer treated with modified radical mastectomy without adjuvant radiation therapy compared with breast-conserving therapy. J Clin Oncol 29: 2852-2858, 2011.

32. Cully M, You H, Levine AJ and Mak TW: Beyond PTEN mutations: The PI3K pathway as an integrator of multiple inputs during tumorigenesis. Nat Rev Cancer 6: 184-192, 2006.

33. López-Knowles E, O'Toole SA, McNeil CM, Millar EK, Qiu MR, Crea P, Daly RJ, Musgrove EA and Sutherland RL: PI3K pathway activation in breast cancer is associated with the basal-like phenotype and cancer-specific mortality. Int J Cancer 126: 1121-1131, 2010. 